\title{
Medical marijuana for urologic chronic pelvic pain
}

\author{
J. Curtis Nickel, MD, FRCSC \\ Department of Urology, Queen's University, Kingston, ON, Canada
}

Cite as: Can Urol Assoc J 2018;12(6Supp|3):S181-3. http://dx.doi.org/10.5489/cuaj.5331

\section{Why marijuana?}

It is generally acknowledged that many patients are not satisfied with the contemporary medical approach to the management of urologic chronic pelvic pain syndrome (UCPPS). Many have turned to marijuana or cannabis because of its strong anecdotal reputation of providing benefit to patients with chronic pain. In a condition in which patients are struggling to cope, the marijuana story appears to offer hope.

\section{What is marijuana?}

Marijuana or cannabis contains hundreds of known compounds (421 chemicals and counting), including cannabinoids (more than 60). Delta 9-tetrahydrocannibinol (THC) and cannabidiol (CBD) are the most widely studied to date. THC (the psychoactive cannabinoid) stimulates appetite, reduces post-traumatic stress disease (PTSD) symptoms, and can be used as a sleep aid. CBD (the non-psychoactive cannabinoid) reduces inflammation, relieves anxiety, and reduces seizures. The combination of CBD and THC may act as a muscle relaxant, relieve spasms, reduce nausea, and relieve pain. At least that is the theory.

\section{Painful lessons learned from the UCPPS clinic}

We have been prescribing medical marijuana for a number of years and are slowly figuring out how to do this properly. We had no textbook, no manual, and no instructions. The literature did not help us. It was really trial and error, with our patients teaching us the optimal strategies for the use of marijuana in UCPPS. Herein, I share the seven most important lessons we have learned.

\section{Lesson 1: Basic research supports the theoretical use of marijuana}

If you prescribe marijuana for your patients with UCPPS, you can be reassured that there are many potential theoretical mechanistic pathways by which marijuana has shown possibilities for benefit in basic science research. These include theoretical analgesic, antiemetic, antispasmodic, anti-inflammatory, antibacterial, relaxant, and anxiolytic properties.

Lesson 2: Clinical research does not provide strong support for the use of marijuana in UCPPS

Unfortunately, there are sparse clinical research studies to support the use of cannabis in UCPPS. In fact, there is almost none and we have to rely on what little clinical evidence is available for the use of marijuana in other chronic pain syndromes. There is at least some literature attesting to the possible benefits of medical marijuana in spastic disorders, PTSD, irritable bowel syndrome, rheumatic diseases, anxiety, depression, sleep disorders, inflammation, and chronic pain. Unfortunately, there is just no solid, long-term data for UCPPS management. The available literature suggests that marijuana can reduce pain by $37 \%$, or a total of 0.5 points on a pain scale of $0-10$. Inhaled cannabis results in short-term reductions in chronic neuropathic pain for one in every 5-6 patients treated. But the use of medical marijuana is also associated with known adverse side effects, which include dizziness, dry mouth, nausea, fatigue, somnolence, euphoria, vomiting, disorientation, drowsiness, confusion, loss of balance, and hallucination.

\section{Lesson 3: Marijuana is better than opioids}

For patients who are having trouble coping because of pain that has not responded to the standard therapies outlined in this supplement, turning to opioids as the last resort is not usually the best approach. Opioids, at best, offer around a $30 \%$ improvement in pain and at worse, offer a paradoxical slow exacerbation in pain intensity. With even minor pain relief comes the possibility of physical or at least psychological dependence to opioids, with desire for further increasing doses with diminished returns. Marijuana has fewer downsides, with the possibility of similar pain relief, better psychological coping, and less chance for addiction and dose escalation. 


\section{Lesson 4: Recognize patients at risk}

All patients with UCPPS are not candidates for medical marijuana management. Patients with a history of substance abuse, diversion risk, and mood disorders should never be prescribed cannabis as a treatment option. If they decide to use the substance on their own, then it will not be a medical error in judgement, but rather a patient-only decision.

\section{Lesson 5: Patient education is the key to successful use of medical} marijuana

Patient education should provide a clear understanding of the benefits (30-40\% reduction in pain) and risks of medical marijuana (Table 1). The different modes of administration (oral, smoking, vaporization), must be a part of the teaching process. The advantages and disadvantages of the various routes of administration are described in Table 2 .

\section{Lesson 6: Dosing considerations}

To find the correct dose for each individual patient, use the adage, "start low and go slow." Do not start immediately with high-dose THC, and for some cautious patients, consider starting only with CBD. For most patients, start with a $\mathrm{TCH} / \mathrm{CBD}$ combination (suggest a 1:1 ratio). It is alright for the patient to experiment with dosing. The averages smoking dose is $1-3 \mathrm{~g} /$ day, with vaporization doses similar. With edibles, a $10 \mathrm{mg}$ dose of either TCH or CBD is reasonable and can be titrated in increments of $5 \mathrm{mg}$ until desired effects. It is probably best to start with the patients making their own edibles to control the concentration. Oil is the most potent form of cannabis. Always start low and rarely ever go to 1 full g/day ( $1 \mathrm{~g}$ of dried cannabis $=6 \mathrm{ml}$ cannabis oil). Consider starting with a single combination THC/CBD capsule and titrate up.

\begin{tabular}{cc}
\hline Table 1. Discussion elements of patient education \\
\hline Risks & Dizziness \\
& Dry mouth \\
& Nausea \\
Fatigue & Somnolence \\
& Euphoria \\
& Vomiting \\
& Disorientation \\
& Drowsiness \\
& Confusion \\
& Loss of balance \\
& Hallucination \\
& $30-40 \%$ reduction in pain \\
Benefit & Oral \\
Mode of administration & Smoking \\
& Vaporization \\
\hline
\end{tabular}

\begin{tabular}{l} 
Table 2. Routes of marijuana administration \\
\hline Smoking \\
Quick action \\
Most common route of administration \\
Least effective for dosing \\
All the crap into lungs \\
Most expensive \\
Preferred over smoking \\
Vaporization \\
5 minutes until into blood stream \\
$10-30$ minutes for full effect \\
Effect duration $2-4$ hours \\
Preferred by females \\
Also available in capsule form \\
$60-90$ minutes for blood stream \\
2 hours for full effect \\
Effect duration 6-8 hours \\
$30-120$ minutes to get into system \\
$2-4$ hours for full effect \\
Duration of effect $8-12$ hours \\
Can be made into a tea
\end{tabular}

\section{Lesson 7: Patient followup is mandatory}

If you prescribe medical marijuana, you are committed to carefully follow up with the patient. The following questions should be addressed and documented at each visit.

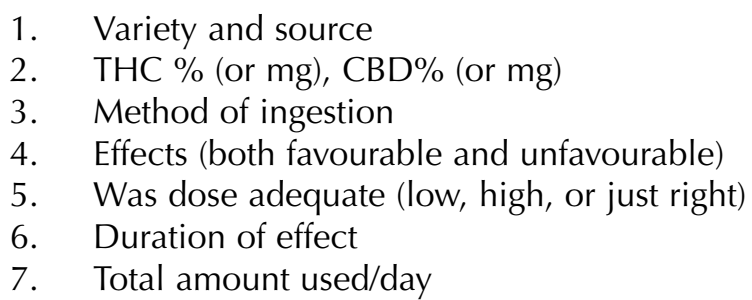

\section{Does a strategy employing medical marijuana benefit our patients with UCPPS?}

Research from both Canadian and international studies have indicated that cannabis treatment may not be the ideal drug for pain relief, but it does seem to result in, at least, a wonderful placebo effect. It is further suggested that cannabis may help our patients cope with their chronic pain syndrome. Since coping with chronic pain is the key to improved quality of life and increased activities in a patient population where medical therapy may fail them, it seems reasonable to consider marijuana treatment as part of a carefully considered, multimodal, biomedical management strategy. Fig. 1 outlines the author's management algorithm.

Competing interests: Dr. Nickel has been a consultant for Astellas, Auxillium, Eli Lilly, Farr Labs, Ferring, GSK, Pfizer, Redleaf Pharma, Taris Biomedical, Tribute, and Trillium Therapeutics; a lecturer for Astellas and Eli Lilly; and has participated in clinical trials supported by Eli Lilly, GSK, J\&J, Pfizer, and Taris Biomedical. 


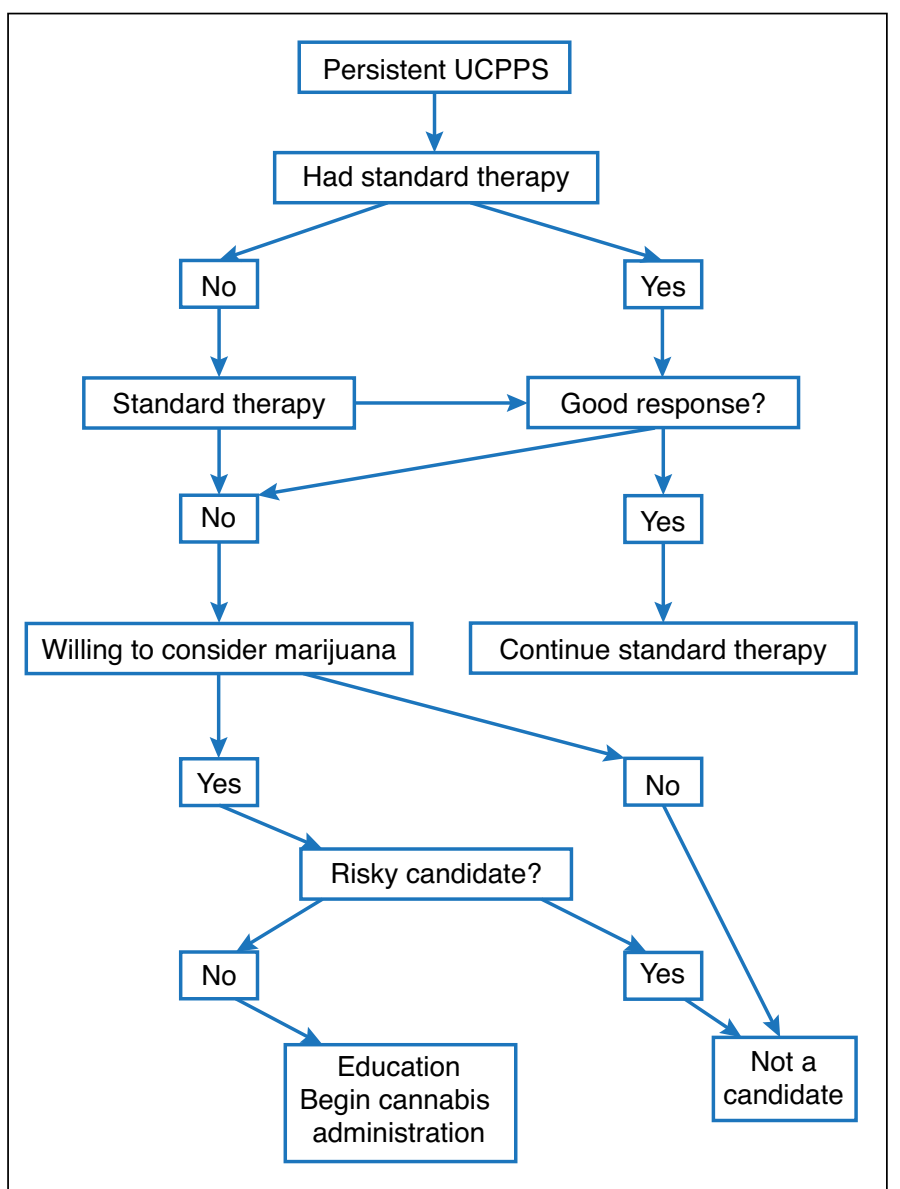

Fig. 1. Urologic chronic pelvic pain syndrome (UCPPS) management algorithm.
This paper has been peer reviewed.

\section{Recommended reading}

1. Tripp DA, Nickel JC, Katz L, et al. Cannabis (marijuana) use in men with chronic prostatitis/ chronic pelvic pain syndrome. Can Urol Assoc J 2014;8:E901-5. https://doi.org/10.5489/cuai.2268

2. Whiting PF, Wolff RF, Deshpande $S$, et al. Cannabinoids for medical use: A systematic review and metaanalysis. JAMA 2015;313:2456-73. https://doi.org/10.1001/jama.2015.6358

3. Andreae MH, Carter GM, Shaparin N, et al. Inhaled cannabis for chronic neuropathic pain: A meta-analysis of individual patient data. J Pain 2015;16:1221-32. https://doi.org/10.1016/i.jpain.2015.07.009

Correspondence: Dr. J. Curtis Nickel, Department of Urology, Queen's University, Kingston, ON, Canada; jin@queensu.ca 